\title{
Arachnoid cyst of the cavum velum interpositum associated with cavum septum pellucidum and cavum vergae
}

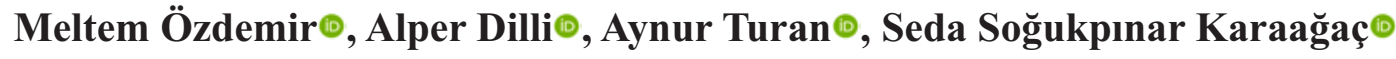 \\ Department of Radiology, Dışkapı Yıldırım Beyazıt Training and Research Hospital, Ankara, Turkey
}

DOI: 10.18621 /eurj.416187

\begin{abstract}
A cavum velum interpositum is a normal variaton which is often detected incidentally in asymptomatic individuals. However, arachnoid cyst of the cavum velum interpositum is extremely rare and may cause symptoms similar to those of a third ventricular mass. On imaging, the main differentials of this cyst are; cystic dilatation of the cavum velum interpositum, cavum vergae cyst, arachnoid cyst of the quadrigeminal cistern, and epidermoid cyst. We present a case of an arachnoid cyst of the cavum velum interpositum coincidentally associated with the cavum septum pellusidum and cavum vergae. The cavum velum interpositum is a rare location for the arachnoid cysts. Accurate diagnosis of the arachnoid cysts of the cavum velum interpositum is important as they cause serious symptoms which can be reversed by surgical treatment.
\end{abstract}

Keywords: Arachnoid cyst, cavum velum interpositum, cavum septum pellucidum, cavum vergae

Received: April 17, 2018; Accepted: April 30, 2019; Published Online: June 30, 2019

$\mathrm{T}$ he cavum septum pellucidum (CSP), along with the cavum vergae $(\mathrm{CV})$ is a persistence of the embryological fluid-filled space between the leaflets of the septum pellucidum and is a common anatomical variant. The CSP and CV normally close in sixth intrauterine week, but may persist in $30 \%$ of term infants and $15 \%$ of adults [1]. The cavum velum interpositum $[\mathrm{CVI}]$, a normal variation, is a dilated cerebrospinal fluid-filled space involving the velum interpositum. It extends below the splenium of the corpus callosum and the column of fornix and above the internal cerebral veins. This variation is often detected incidentally in asymptomatic individuals [2]. However, arachnoid cyst of the CVI is extremely rare and may cause symptoms similar to those seen with a third ventricular mass [3]. We report a case of an arachnoid cyst of the CVI coincidentally associated with CSP and CV, who presented with headache and memory disturbance.

\section{CASE PRESENTATION}

A 64-year-old man with complaints of headache and memory disturbance for three months, was referred to the Department of Radiology for a brain magnetic resonance imaging (MRI). His neurological examination findings were unremarkable. MRI study revealed CSP and CV. There was a $48 \times 42 \times 24 \mathrm{~mm}$ sized, thin-walled cystic lesion lying just posterior to the CV (Figure 1). The cyst was located between the posterior halves of the lateral ventricles and the roof of the third ventricle (Figure 2). It was isointense with the cerebrospinal fluid in all pulse sequences (Figure 3 ). By the compressive effect of the cyst; posterior half of the corpus callosum was elevated, the fornices were splayed and the internal cerebral veins were inferiorly displaced. The lateral ventricles were mildly dilated but the quadrigeminal cistern was normal (Figures 2

Address for correspondence: Meltem Özdemir, MD., Dışkapı Yıldırım Beyazıt Training and Research Hospital, Department Radiology, Ankara, Turkey E-mail: meltemgu@yahoo.com 


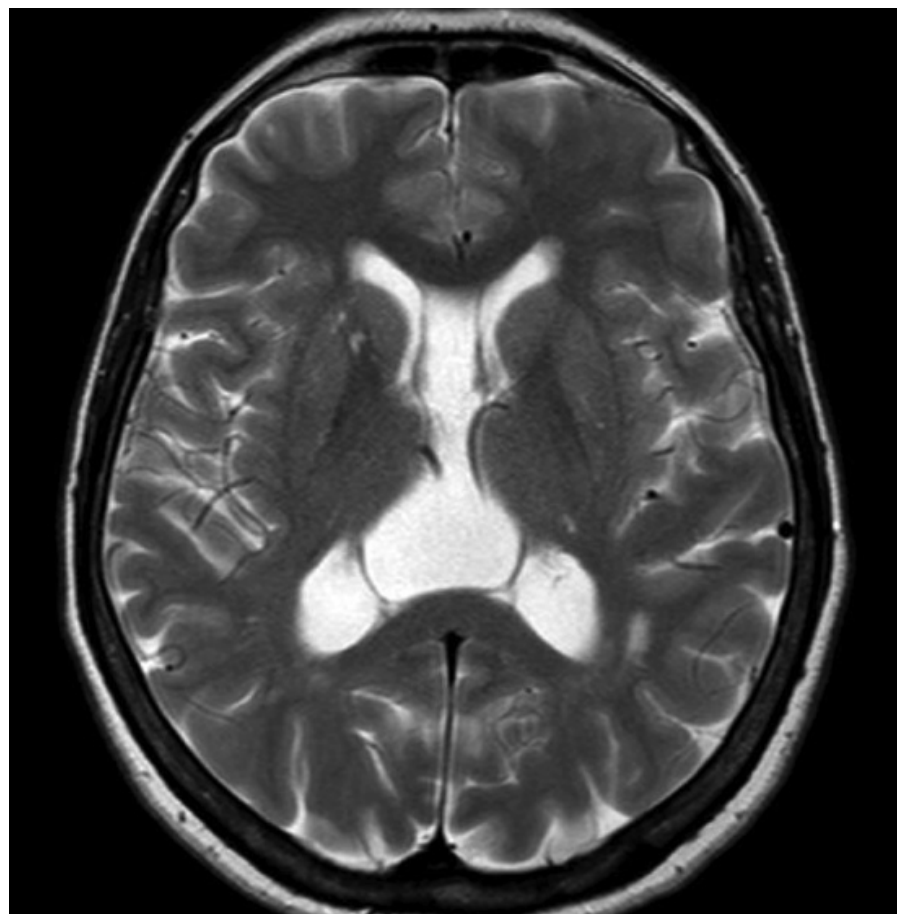

Figure 1. Axial T2-weighted section through lateral ventricles shows cavum septum pellucidum and cavum vergae. An arachnoid cyst of the cavum velum interpositum, of which the anterior border is invisible in this section, is also present.

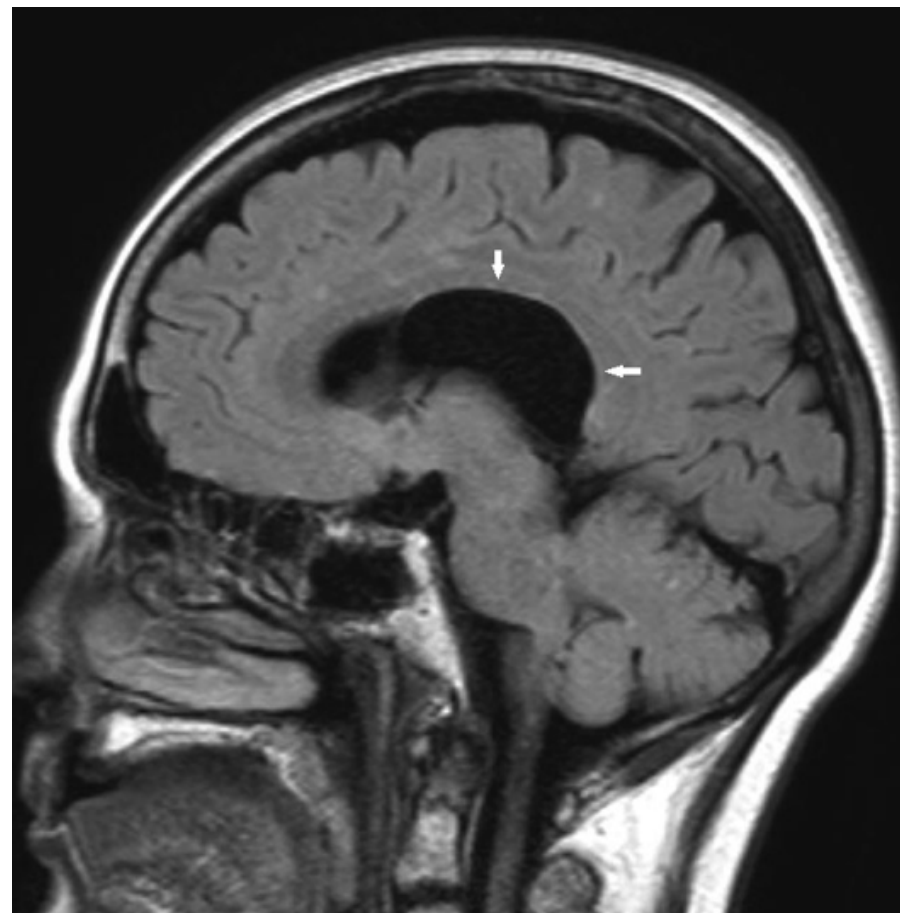

Figure 2. Sagittal FLAIR image reveals a large cystic lesion located in the cavum velum interpositum. The posterior half of the corpus callosum is elevated, the splenium is slightly depressed (arrows). Note the quadrigeminal cistern is normal.
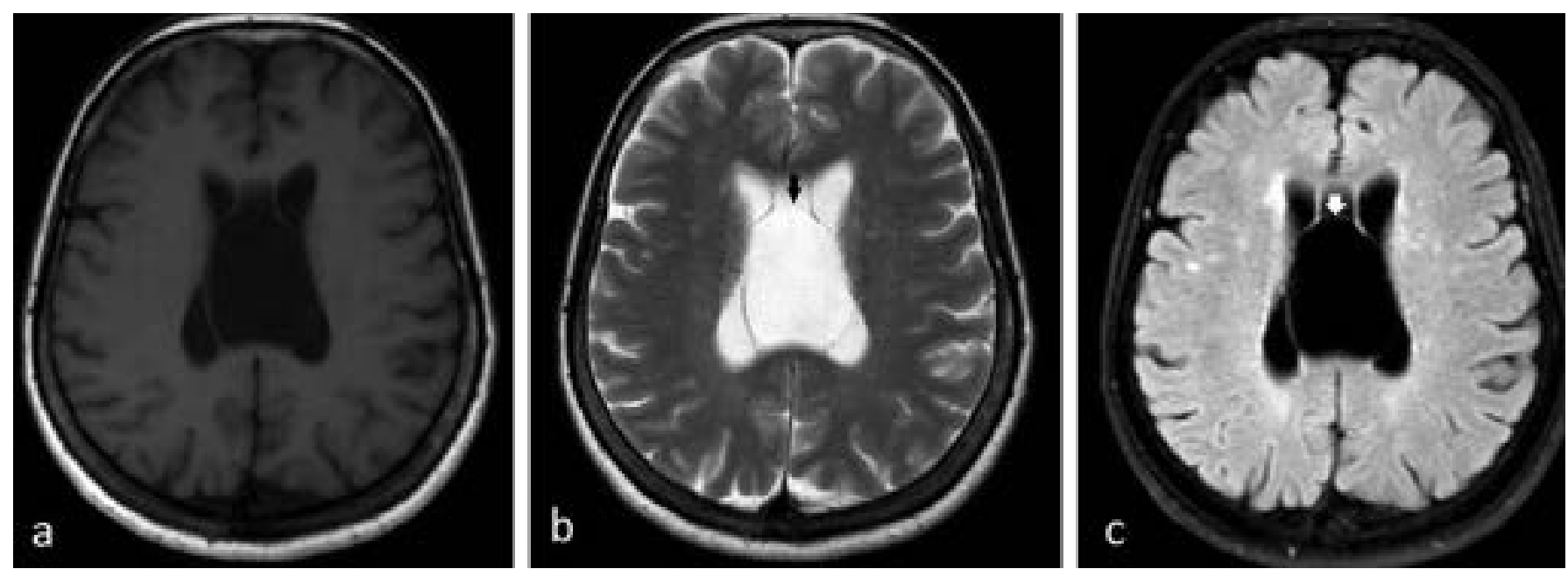

Figure 3. The cyst is isointense with the cerebrospinal fluid in T1-weighted [a], T2-weighted [b], and FLAIR [c] sequences. The anterior wall of the cyst is seen in $\mathrm{b}$ and $\mathrm{c}$ [arrows]. Note the slight dilatation of the lateral ventricles.

and 4). On diffusion weighted images, no restriction of diffusion was revealed (Figure 5). By these imaging findings, the patient was diagnosed as having an arachnoid cyst of the CVI.

\section{DISCUSSION}

The CSP is regarded as a part of the longitudinal cerebral fissure, which becomes walled off by the union of the hemispheres forming the corpus callosum above and the fornix below. The cerebrospinal fluidfilled space between the septa pellucida, two paired clear membranes, is a cavity of which the anterior part is called CSP, whereas the posterior part is called CV [4]. During development, these spaces obliterate antero-posteriorly: the CV followed by the CSP, and it is common that both occur together. This variation is often detected incidentally and individuals are usually asymptomatic, however, there are some 


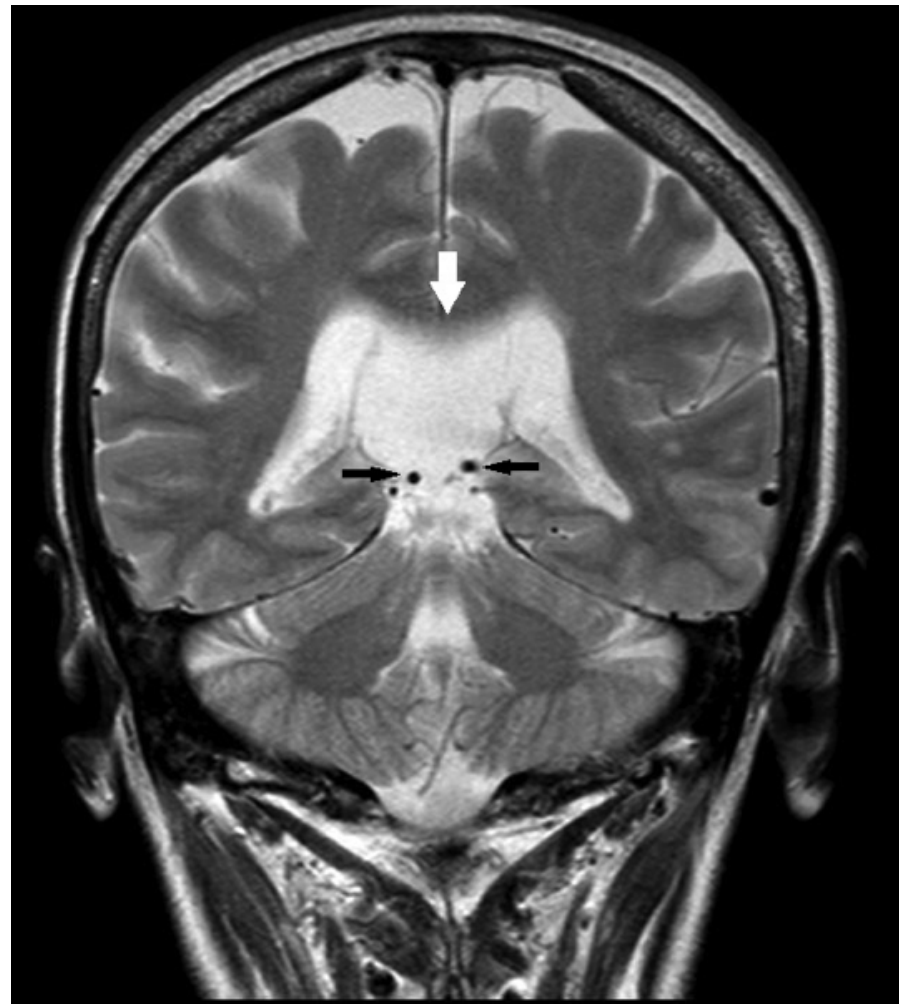

Figure 4. Coronal T2-weighted image demonstrates a large cyst between the lateral ventricles and above the third ventricle (white arrow). Internal cerebral veins are displaced inferiorly (black arrows). The lateral ventricles are slightly dilated.

studies reporting an association between the CSP and neuropsychiatric disorders [5].

CVI is a potential space which is actually a forward extension of the quadrigeminal cistern. It is located below the splenium of the corpus callosum and the column of fornix, and above the internal cerebral veins. It has a triangular shape of which the apex anterorly reaches the foramina of Monro [2]. A CVI is a frequent finding among infants and young children, and it is supposed to close by aging. If not closed, it may be detected in adulthood, mostly as an incidental asymptomatic finding [6].

Arachnoid cyst of CVI is extremely rare. To the best of our knowledge, to date, there have only seven cases of arachnoid cyst of this location been reported [3, 7-12]. The probable origin of the arachnoid cyst of CVI is thought to be tela choroidea, a vascular connective tissue which lies in the space between the lateral and third ventricles [12]. It can cause similar symptoms of a third ventricular mass [3, 8]. Furthermore, an intracranial arachnoid cyst can cause reduced perfusion and metabolism in the surrounding cortical regions [9]. These changes can result in mental impairments including memory disturbances as they do in the present case. In the previous cases, the presenting symptoms of the arachnoid cyst of the CVI were reported to be; headache, loss of consciousness, dizziness, disorientation, confusion, and memory disturbances. Our patient presented with headache and memory impairment.

The imaging characteristic of an arachnoid cyst of the CVI is a midline cyst located between the third ventricle and the posterior halves of the lateral ventricles which causes elevation and splaying of the fornices and inferiorly displacing the internal cerebral veins [3]. On imaging, the main differentials of this cyst are; cystic dilatation of the CVI, CV cyst, arachnoid cyst of the quadrigeminal cistern, and epidermoid cyst. The major characteristic of the cystic dilatation of the CVI is that it has free communication with the quadrigeminal cistern, thus it typically does
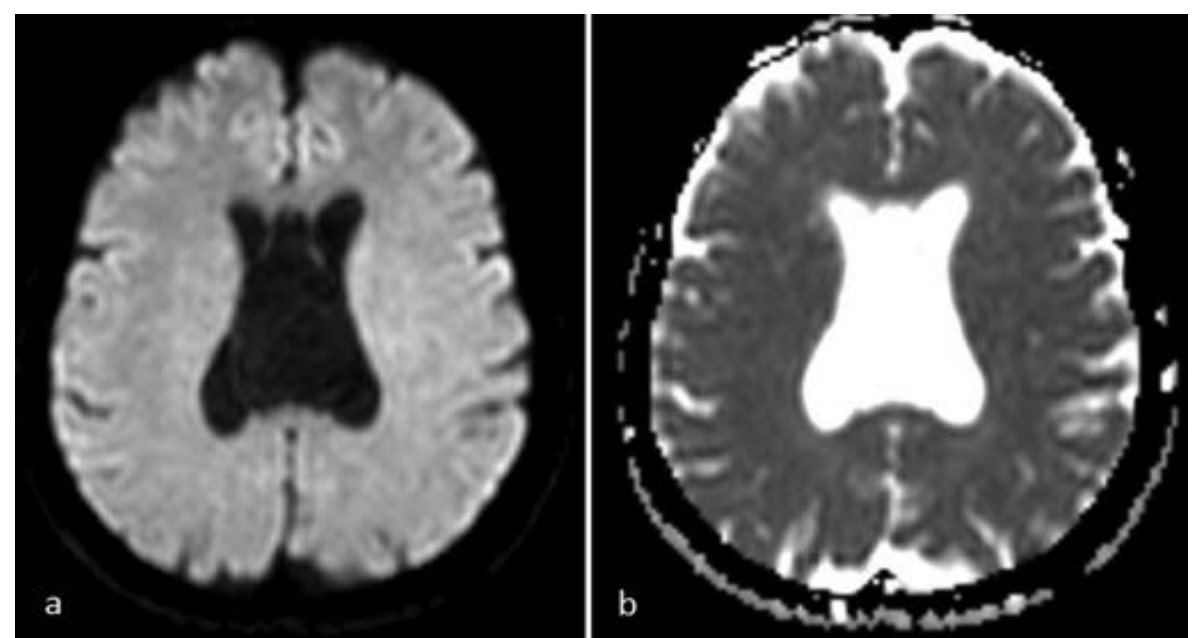

Figure 5. No restriction of diffusion is noted in diffusion weighted images (a) and ADC mapping (b). 
not cause mass effect or hydrocephalus [9]. However, as in the present case, an arachnoid cyst is prone to obstruct the ventricles causing symptoms closely similar to those of a third ventricular mass. To differentiate a CV cyst from an arachnoid cyst of CVI, the internal cerebral veins are used as a landmark. As $\mathrm{CV}$ lies above the veins, the cyst of the $\mathrm{CV}$ is seen separately from the veins. However, as CVI encloses the veins, an arachnoid cyst of the CVI encloses the veins at its lower and lateral borders [3]. The close relation of the veins and the cyst was clearly seen in our case (Figure 4). An arachnoid cyst originating from the quadrigeminal cistern can mimic the arachnoid cyst of the CVI if it extends in the superoanterior direction. However, different from the arachnoid cyst of the CVI, it displaces the internal cerebral veins upward as arachnoid membrane in the quadrigeminal cistern is topologically below the veins. An epidermoid cyst of any location can easily be differentiated from an arachnoid cyst by diffusion weighted imaging. Epidermoid cysts show diffusion restriction, whereas arachnoid cysts do not $[3,9]$.

Arachnoid cysts of the CVI should be treated if they become symptomatic. A communication between the cyst and the ventricular system is provided by endoscopic ventricular fenestration in order to reduce the mass effect of the large cyst. In the previous cases, most of the symptoms were reported to be reversed by surgical treatment $[7,9,10]$.

\section{CONCLUSION}

The CVI is a rare location for the arachnoid cysts. Accurate diagnosis of the arachnoid cysts of the CVI is important as they cause symptoms similar to those of a third ventricular mass, and these symptoms can be reversed by surgical treatment.

\section{Informed consent}

Written informed consent was obtained from the patient for publication of this case report and any accompanying images.

\section{Conflict of interest}

The authors declared that there are no potential conflicts of interest with respect to the research, authorship, and/or publication of this article.

\section{REFERENCES}

[1] Dähnert W. Differential diagnosis of brain disorders. In: Dähnert W, ed., Radiology Review Manual, 7th ed., Philadelphia: Lippincott Williams \& Wilkins;2011:233.

[2] Ketonen LM, Hiwatashi A, Sidhu R, Westesson PL. Pediatric Brain and Spine. Germany, Springer; 2005:11-2.

[3] Rajesh S, Bhatnagar S, Chauhan U, Gupta S, Agarwal N, Kasana V. Arachnoid cyst of the cavum velum interpositum in a septuagenarian: radiological features and differential diagnosis. Neuroradiol J 2014;27:154-7.

[4] Winter TC, Kennedy AM, Byrne J, Woodward PJ. The cavum septi pellucidi: why is it important? J Ultrasound Med 2010;29:427-44.

[5] Chen CY, Chen FH, Lee CC, Lee KW, Hsiao HS. Sonographic characteristics of the cavum velum interpositum. AJNR Am J Neuroradiol 1998;19:1631-5.

[6] Tong CK, Singhal A, Cochrane DD. Endoscopic fenestration of cavum velum interpositum cysts: a case study of two symptomatic patients. Childs Nerv Syst 2012;28:1261-4.

[7] Galarza M, Merlo AB, Ingratta A, Albanese EF, Albanese AM. Cavum septum pellucidum and its increased prevalence in schizophrenia: a neuroembryological classification. J Neuropsychiatry Clin Neurosci 2004;16:41-6.

[8] Lee YW, Ahn YJ, Huh R, Chae KY. Arachnoid cyst of the velum interpositum: coincidence with multiple cranial neuropathies: case report. J Korean Neurosurg Soc 2002;32:15961.

[9] Funaki T, Makino Y, Arakawa Y, Hojo M, Kunieda T, Takagi $\mathrm{Y}$, et al. Arachnoid cyst of the velum interpositum originating from tela choroidea. Surg Neurol Int 2012;3:120.

[10] Gangemi M, Donati P, Maiuri F, Sigona L. Cyst of the velum interpositum treated by endoscopic fenestration. Surg Neurol 1997;47:134-6; discussion 136-7.

[11] Hartmann TM, Braun SD, Kieffer HL. Arachnoid cyst of the velum interpositum. AJNR Am J Neuroradiol 1989;10:1127.

[12] Spiegel SM, Nixon B, TerBrugge K, Chiu MC, Schutz H. Arachnoid cyst of the velum interpositum. AJNR Am J Neuroradiol 1988;9:981-3. 